\title{
Competência Cognitiva e Resolução de Problemas com Equações Algébricas do $1^{\circ}$ Grau
}

\section{Cognitive Competence and Problem-Solving Involving 1st Degree Algebraic Equations}

\author{
Yasmini Lais Spindler Sperafico* \\ Beatriz Vargas Dorneles ${ }^{* *}$ \\ Clarissa Seligman Golbert ${ }^{* * *}$
}

\begin{abstract}
Resumo
Esta pesquisa investigou a relação entre a competência cognitiva e o desempenho na resolução de problemas com equações algébricas do $1^{\circ}$ grau. 38 indivíduos, de 12 a 15 anos, do $8^{\circ}$ ano de uma escola pública de Porto Alegre, foram agrupados em dois grupos, alto e baixo nível de competência cognitiva, utilizando-se o WASI. Estes estudantes realizaram a Tarefa de Resolução de Problemas com Equações Algébricas do $1^{\circ}$ Grau (TRPEA) para verificarmos a existência de relação entre a competência cognitiva e a resolução de problemas, bem como as diferenças de desempenho entre os grupos. Encontrou-se correlação significativa $(\mathrm{p}<0,001)$ entre o WASI e a TRPEA e diferença significativa $(\mathrm{p}<0,001)$ entre os dois grupos quanto ao desempenho na TRPEA. Os resultados obtidos confirmam achados anteriores, evidenciando a relação entre a competência cognitiva e o desempenho na resolução de problemas com equações algébricas do $1^{\circ}$ grau. Implicações para o ensino escolar são discutidas.
\end{abstract}

Palavras-chave: Competência Cognitiva. Resolução de Problemas. Equações Algébricas do $1^{\circ}$ Grau.

\begin{abstract}
This investigation explored the relationship between cognitive competence and performance in solving problems with 1st degree algebraic equations. Thirty-eight students, aged 12 to 15 , attending the 8th year of primary education in a public school in Porto Alegre, were divided into two groups, according to high or low cognitive competence level, using WASI - Whimbey Analytical Skills Inventory. These students accomplished the Problem Solving 1st Degree Algebraic Equations Task (PSAET) sothe authors could inquire about the existence of connection between cognitive competence and problem solving, as well as to check differences in group performances. The results obtained were: significant correlation $(\mathrm{p}<0,001)$ between WASI and PSAET and

\footnotetext{
* Doutoranda em Educação pela Universidade Federal do Rio Grande do Sul (UFRGS). Bolsista Capes, Universidade Federal do Rio Grande do Sul, Porto Alegre, Brasil. Endereço: Av. Paulo Gama 110, prédio 12201, $7^{\circ}$ andar, sala 700-08, CEP: 90046-060, Porto Alegre, RS, Brasil; E-mail: yasminisperafico@gmail.com.

** Pós-Doutora em Educação pela Universidade de Oxford, Oxford, Inglaterra. Doutora em Educação pela Universidade de São Paulo (USP). Professora do Programa de Pós Graduação em Educação da Faculdade de Educação, Universidade Federal do Rio Grande do Sul (UFRGS), Porto Alegre, RS, Brasil. Endereço: Av. Paulo Gama 110, prédio 12201, $7^{\circ}$ andar, sala 700-08, CEP: 90046-060, Porto Alegre, RS, Brasil; E-mail: bvdornel@terra.com.br.

Doutora em Educação pela Universidade Federal do Rio Grande do Sul (UFRGS). Professora Adjunta do Departamento de Estudos Especializados da Faculdade de Educação, Universidade Federal do Rio Grande do Sul, Porto Alegre, Rio Grande do Sul, Brasil. Endereço: Av. Paulo Gama 110, prédio 12201, $7^{\circ}$ andar, sala 70008, CEP: 90046-060, Porto Alegre, RS, Brasil; E-mail: mcgolbert@uol.com.br.
} 
significant difference $(\mathrm{p}<0,001)$ between two groups in reference to the performance in PSAET. Such results confirm previous findings, emphasizing the relationship between cognitive competence and performance in $1 \mathrm{st}$ degree algebraic equations problem solving. School education implications are discussed.

Keywords: Cognitive Competence. Problem Solving. 1st Degree Algebraic Equations.

\section{Introdução}

Os alunos utilizam estratégias diversas frente a um mesmo problema matemático envolvendo equações algébricas do $1^{\circ}$ grau. Considerando tal diversidade, Davis, Nunes e Nunes (2005) afirmam que encontrar caminhos para a resolução de um problema depende do interesse que o problema desperta em cada um, dos mecanismos que cada um possui para desvendá-lo, dos conhecimentos conceituais e dos recursos cognitivos atuantes. Portanto, como sugere Chi (2011), as diferenças no uso de estratégias estão baseadas em processos cognitivos e organizações mentais que os humanos têm em comum, e que caracterizam sua competência para a solução de problemas.

Nos últimos anos, estudos têm mostrado (PRIMI et al., 2001, PRIMI, 2002; CAMPOS, 2004; SANTOS; PRIMI, 2005; CHI, 2011) a existência da relação entre o desempenho na resolução de problemas específicos e a competência cognitiva, entendida aqui como uma capacidade geral para a resolução de problemas, sendo um fator essencial da aprendizagem, já que parte das dificuldades na resolução de problemas específicos pode ser explicada por baixos níveis de competência cognitiva. Entretanto, essa não é uma relação natural, já que as habilidades cognitivas amplas nem sempre são aplicadas imediatamente pelo aluno para resolver um problema específico (MATLIN, 2004; DAVIS; NUNES; NUNES, 2005; CHI, 2011), como na área da aritmética. Nessa área, a competência em cálculo, ou seja, a capacidade de operar com números, é necessária, mas não suficiente para o bom desempenho na resolução de problemas algébricos. Isso porque são necessárias, além de competências em cálculo, também competências cognitivas, para compreender o problema e tomar decisões apropriadas quanto ao uso de estratégias para a sua resolução (MAYER, 1992). Portanto, essa relação entre competência geral e desempenho específico, observada na área da aritmética, não pode ser generalizada para outras áreas matemáticas sem maiores investigações quanto à veracidade dessa relação.

Sendo assim, há necessidade de pesquisas que investiguem a relação entre competência cognitiva e resolução de problemas em outros campos específicos, como o algébrico, principalmente no que se refere às equações do $1^{\circ}$ grau, já que os estudos envolvendo esse importante componente da álgebra ainda são escassos. Desse modo, o 
presente estudo teve como objetivo investigar a existência dessa relação, apontada na literatura, entre o desempenho na resolução de problemas específicos, nesse caso, problemas com equações algébricas do $1^{\circ}$ grau e uma competência cognitiva geral para a resolução de problemas, bem como descrever as diferenças de desempenho entre estudantes com alto (ACC) e baixo (BCC) nível de competência cognitiva e discutir as implicações da existência (ou não) dessa relação para o ensino do conteúdo em questão. Optou-se pelo campo algébrico por esse marcar uma importante ruptura no ensino da Matemática, de uma Matemática supostamente concreta para um campo mais abstrato e generalizável (VERGNAUD, 1996), sendo as equações do $1^{\circ}$ grau um dos primeiros conteúdos algébricos formalizados que os alunos têm contato.

Para isso, inicialmente, apresenta-se uma breve discussão sobre o campo algébrico e a resolução de problemas envolvendo equações algébricas do $1^{\circ}$ grau, com o aporte de estudiosos dessa área da Matemática (BOOTH, 1995; DA PONTE; BRANCO; MATOS, 2009). A seguir, discute-se a competência cognitiva e sua definição, na perspectiva da Psicologia Cognitiva, bem como sua relação com a resolução de problemas, a qual tem sido evidenciada em diversos estudos (CHI; GLASER, 1992; NOKES; SCHUNN; CHI, 2010; CHI, 2011). Após, apresenta-se o método adotado na presente pesquisa, discute-se os resultados obtidos, tecendo algumas conclusões parciais e implicações para o ensino escolar.

\section{Resolução de problemas com equações algébricas do $1^{\circ}$ grau}

A álgebra é um ramo da Matemática que se ocupa da simbolização de relações numéricas, de estruturas matemáticas e das operações sobre essas estruturas. Seu foco incide no estabelecimento de procedimentos e relações, numa forma simplificada geral. Assim, a razão de se estabelecer essas relações gerais é usá-las como regras de procedimento para a resolução adequada de problemas, encontrando respostas numéricas. Entretanto, Booth (1995) afirma que o foco inicial do ensino de álgebra é o estabelecimento, a expressão e a manipulação de afirmações gerais. Dessa forma, o estudo da álgebra consiste de um espaço bastante significativo para que o aluno desenvolva e exercite sua capacidade de abstração e generalização e, além disso, lhe possibilita a aquisição de uma poderosa ferramenta para resolver problemas (BOOTH, 1995; BERNARD; COHEN, 1995).

Um dos primeiros conteúdos algébricos apresentado aos alunos é a resolução de equações do $1^{\text {o }}$ grau. Essas, na perspectiva de Bernard e Cohen (1995), desempenham um 
papel muito importante na Matemática e em muitas de suas aplicações, sendo um elemento essencial no ensino da álgebra, já que possibilita a representação e solução de problemas mais complexos que não poderiam ser resolvidos apenas com recursos aritméticos. Uma equação é caracterizada pela existência de letras indicando valores desconhecidos, que são denominadas incógnitas, um sinal de igualdade, uma expressão à esquerda da igualdade, denominada primeiro membro, e uma expressão à direita da igualdade, denominada segundo membro. Os membros de uma equação, por sua vez, são compostos por termos, que são informações numéricas ou incógnitas.

O trabalho com equações pressupõe a familiarização dos discentes com essas terminologias, como alertam Da Ponte, Branco e Matos (2009). Os autores ainda destacam a importância da noção de solução de uma equação (raiz), pois, além de serem capazes de resolver equações, os alunos devem ser capazes de verificar se um dado valor é ou não solução de certa equação. A importância do conhecimento pelo educando da definição de raiz também é destacada por Bernard e Cohen (1995), sendo considerada pelos autores como marco inicial de um estudo significativo sobre a resolução de equações. A raiz de uma equação é valor que a torna verdadeira, ou seja, que valida a igualdade entre o primeiro e o segundo membro.

Uma equação do $1^{\circ}$ grau pode ser solucionada de diferentes maneiras. Bernard e Cohen (1995) destacam quatro métodos de solução, os quais podem constituir também uma sequência de ensino evolutiva, desde métodos mais simples e vagos, a métodos mais complexos. Os métodos, na sequência de aprendizagem são: (1) gerar e avaliar, (2) esconder, (3) desfazer e (4) equações equivalentes.

O método de gerar e avaliar consiste em gerar valores aleatórios para a incógnita e aplicá-los à equação verificando ou não a validade. O método de esconder é aplicado na resolução de equações simples, consistindo em esconder a incógnita e fixar a atenção ao que a equação pede. Assim, na situação $15-\mathrm{x}=12$, esconder-se-ia a incógnita $\mathrm{x}$ e se perguntaria quinze menos quanto resulta em 12? Já o método de desfazer baseia-se nas noções de inversos operacionais e na reversibilidade de um processo envolvendo um ou mais passos invertíveis. Desse modo, as operações, geralmente do primeiro membro, são desfeitas, por meio de operações inversas, buscando isolar a incógnita e determinar seu valor.

O último e mais complexo método de resolução de equações pressupõe a compreensão do conceito de equações equivalentes. Para isso, primeiramente deve haver uma compreensão mais profunda do sinal de igualdade, que deve deixar de pressupor um resultado, como 
frequentemente é compreendido pelo aluno, e passar a representar a existência de equivalência. Assim o método de equações equivalentes é semelhante ao método de desfazer, mas pelo fato da equação constituir uma equivalência, as operações devem ser desfeitas em ambos os membros da equação (BOOTH, L., 1995; BOOTH, J.; KOEDINGER, 2008; GINSBURG, 2009).

A aprendizagem sobre a resolução de equações parece ser mais eficiente no contexto de resolução de problemas (BERNARD; COHEN, 1995). Para os autores, o contexto de resolução de problemas contribuiria no desenvolvimento de processos para eliminar obstáculos e atingir subobjetivos, criando assim, meios para monitorar e avaliar processos, dando ênfase à tarefa e sua realização bem-sucedida.

Posto isso, para resolver problemas com equações algébricas do $1^{\circ}$ grau, os estudantes necessitam de conhecimentos aritméticos anteriormente aprendidos (operações aritméticas), de conhecimentos sobre terminologias utilizadas nos problemas algébricos (incógnita, membro, termo, raiz), assim como outras habilidades cognitivas que constituem a competência cognitiva para a resolução de problemas (raciocínio, capacidade de processamento cognitivo, criatividade), que serão melhor descritas posteriormente.

Caracterizado o campo específico de resolução de problemas abordado neste estudo, passamos a discorrer sobre a competência cognitiva, sua definição e relação com a resolução de problemas matemáticos específicos.

\section{Competência Cognitiva}

A competência cognitiva para a resolução de problemas pode ser definida como a capacidade de um indivíduo de se envolver em processamento cognitivo para compreender e resolver problemas em que um método de solução não é imediatamente óbvio, e inclui a vontade de se envolver com problemas, com o objetivo de alcançar seu potencial como cidadão construtivo e reflexivo. Essa definição de competência cognitiva, sob o enfoque da Psicologia Cognitiva, parece envolver muito mais do que a reprodução do conhecimento básico acumulado. Trata-se de uma mobilização de aptidões cognitivas e práticas, habilidades criativas, entre outros fatores cognitivos (TARDIF, 2003; OEDC, 2010).

Nessa perspectiva, a competência cognitiva não corresponde a uma forma de algoritmo memorizado e praticado repetidamente, visando à garantia de sustentabilidade e replicabilidade, mas uma forma de agir muito flexível e adaptável a vários contextos e para 
diferentes problemas, que é passível de ser aprendida (TARDIF, 2003). Assim, para Primi et al. (2001), dizer que um sujeito é competente significa dizer que este atingiu determinado nível de realização. Essa realização se faz possível por meio do uso de estratégias cognitivas e metacognitivas eficazes e bem aplicadas na resolução de problemas (GULGOZ; KAGITCIBASI, 2004).

Dessa forma, a definição de competência cognitiva apresentada por Tardif (2003) e adotada pelo documento do Pisa (OEDC, 2010) contempla o raciocínio, capacidade de processamento cognitivo e criatividade e ainda destaca a importância do conhecimento prévio utilizado pelo sujeito ao se deparar com um problema novo. Essa definição, na perspectiva de Primi et al.(2001) é melhor formulada que as definições anteriores, já que relaciona os conceitos de habilidade, conteúdo e nível de realização, indispensáveis para a resolução adequada de um problema matemático específico.

\subsection{Competência cognitiva na resolução de problemas}

Alunos com alto nível de competência cognitiva são aqueles com maior capacidade de organização dos conhecimentos conceitual e procedural na memória de longo prazo, podendo, dessa forma, acessá-los mais facilmente durante a resolução de um problema. Para que isso seja possível, estes estudantes utilizam estratégias para aprimorar seus recursos mnemônicos. Esses indivíduos também processam melhor as informações envolvidas na resolução do problema, já que automatizaram várias operações por meio da prática na aplicação de estratégias, podendo recuperá-las e executá-las com facilidade (CHI; GLASER, 1992; NOKES; SCHUNN; CHI, 2010; CHI, 2011).

Além disso, como indicado por Nokes, Schunn e Chi (2010), estes alunos utilizam processos de esquematização, desenvolvendo esquemas ricos e bem organizados, e processos de automatização de sequências de passos em rotinas que exigem pouco controle consciente. Por meio desses processos, eles podem redimensionar o peso de solucionar o problema da memória de trabalho, de capacidade limitada, para a memória de longo prazo, de capacidade muito maior, permitindo-lhes, assim, monitorar melhor seu avanço e sua precisão durante a resolução. Matlin (2004) ainda destaca que alunos mais competentes utilizam mais a metacognição, o que lhes permite avaliar a dificuldade do problema e torna-os também mais capazes de reconhecer os erros que cometem e distribuir adequadamente o tempo ao desenvolverem a resolução. 
Esses estudantes mais competentes também apresentam maior conhecimento dos elementos dos problemas, das operações possíveis de serem realizadas e da meta a ser atingida. E, por dispor de maior e mais organizado conhecimento, desenvolvem uma melhor representação do problema e escolhem a estratégia de resolução mais eficiente (CHI, 2011).

Diversos estudos têm indicado essa relação entre competência cognitiva e desempenho em problemas específicos, como os que envolvem jogos de xadrez (DE GROOT, 1966; CHASE; SIMON, 1973) ou quebra-cabeças (CHI; GLASER, 1992); e problemas em domínios acadêmicos específicos, como física (CHI; FELTOVICH; GLASER, 1981; DUFRESNE et al., 1992; CHI; ROY; HAUSMANN, 2008) e aritmética (MAYER, 1992).

Em resumo, parece haver evidências quanto à existência de uma relação entre a competência cognitiva geral para a resolução de problemas e o desempenho na resolução de problemas específicos. Entretanto, verificou-se uma inexistência de pesquisas que investiguem essa relação no contexto de problemas algébricos com equações do $1^{\circ}$ grau. Dessa forma, o presente estudo teve como objetivo verificar a validade dessa relação descrita pela literatura nesse campo específico da resolução de problemas matemáticos, discutindo suas implicações para o ensino desse conteúdo específico do campo algébrico.

\section{Método}

O estudo correlacional envolveu 38 estudantes brasileiros, 16 meninos e 22 meninas, $(\mathrm{IM}=12,7$ anos; $\mathrm{DP}=0,66)$, alunos do $8^{\circ}$ ano de uma escola pública da região metropolitana de Porto Alegre - RS. Definiu-se, como critérios de exclusão, frequência inferior a $75 \%$ e percentil inferior a 20 no Teste Não Verbal de Inteligência (R-1).

O Whimbey Analytical Skills Inventory, WASI, (WHIMBEY; LOCHHEAD, 1999), em versão adaptada (SPERAFICO, 2013), avaliou a competência cognitiva e permitiu separar os estudantes em dois grupos, alto (ACC) e baixo (BCC) nível de competência cognitiva, de acordo com a pontuação geral. Para verificar o desempenho dos estudantes nos problemas com equações algébricas do $1^{\mathrm{o}}$ grau, aplicou-se a Tarefa de Resolução de Problemas com Equações Algébricas do $1^{\circ}$ grau (TRPEA), composta por quatro problemas verbais com mesmo nível de complexidade, adaptados de Dante (2007). Para identificar a existência de correlação entre as funções investigadas e a diferença entre os grupos ACC e BCC, aplicou-se o teste de Correlação de Pearson e o teste t-Student, respectivamente. 


\subsection{Descrição do Whimbey Analytical Skills Inventory (WASI)}

O WASI foi desenvolvido por Arthur Whimbey e publicado em seu livro Problem Solving and Comprehension, em 1944, em colaboração com Jack Lochhead, com reedições posteriores. Esse instrumento tem sido utilizado em diversos estudos como recurso para avaliar a competência cognitiva (SANLEY, 1987; REINERT, 1989; VIEIRA, 1999; GULGOZ; KAGITCIBASI, 2004). O WASI é formado por um pré e um pós-teste, com 38 problemas cada, distribuídos em seis categorias distintas: 1) Problemas de Raciocínio Verbal, 2) Problemas de Instruções Sequenciais, 3) Problemas de Formação de Analogias, 4) Problemas de Análise de Tendências e Padrões, 5) Problemas de Resolução Matemática e 6) Problemas de Relação de Frases Escritas. Neste estudo, adotou-se apenas o pré-teste, em versão traduzida, que foi utilizado no estudo de Vieira (1999). O WASI foi aplicado em momento coletivo.

Por meio de um estudo dos problemas presentes no instrumento e aplicação do mesmo em pesquisa piloto anterior (SPERAFICO, 2013), reduziu-se a prova a vinte e dois problemas, retirando os problemas que apresentaram algum obstáculo aos estudantes quanto à compreensão da linguagem, bem como possuíam elevado nível de dificuldade, medido por meio do número de acertos (os problemas com ausência de acertos foram retirados). Entretanto, procurou-se manter a proporcionalidade de questões em cada categoria, exceto a de problemas de resolução matemática, pois foram mantidos os dois problemas existentes, uma vez que o estudo está mais voltado a essa área do conhecimento.

\subsection{Descrição da Tarefa de Resolução de Problemas com Equações Algébricas do $1^{\circ}$ Grau (TRPEA)}

A Tarefa de Resolução de Problemas com Equações Algébricas do $1^{\circ}$ grau (TRPEA) é composta por quatro situações-problema que apresentam o mesmo nível de dificuldade, adaptadas de Dante (2007). Os alunos foram solicitados a resolver os problemas individualmente, mas podiam pedir auxílio ao pesquisador para compreender a situaçãoproblema. O auxílio era proporcionado por meio de questionamentos dirigidos aos estudantes, com o objetivo de auxiliá-lo na representação do problema em questão.

A aplicação da TRPEA foi realizada pela pesquisadora, em espaço reservado disponibilizado pela escola, durante o turno escolar. O instrumento teve tempo médio de 
aplicação de 28 minutos e 20 segundos. Antes de iniciar a tarefa, realizou-se a resolução do problema de ensaio: $O$ dobro da idade de Taís somado a 30 é o mesmo que sua idade acrescida de 50. Qual a idade de Taís? Com base neste problema, o estudante foi questionado sobre seus conhecimentos em relação a equações e ao procedimento adotado por ele para a resolução. Os problemas que compuseram a TRPEA são apresentados no Quadro 1:

\section{Tarefa de Resolução de Problemas com Equações Algébricas do $1^{\circ}$ Grau (TRPEA):}

1. Carlos estava realizando cálculos envolvendo o valor de sua idade e percebeu que subtraindo 13 anos do dobro de sua idade obtinha-se o mesmo que sua idade acrescida de 5 anos. Qual é a idade de Carlos?

2. Ângela e sua amiga Carina colecionam figurinhas. As amigas estavam comparando suas quantidades quando Ângela sugeriu que se quadruplicasse sua quantidade de figurinhas e do resultado subtraí-se 12, teria a mesma quantidade que o dobro de figurinhas de Carina acrescido de 36. Sabendo que elas possuíam a mesma quantidade de figurinhas, quantas figurinhas tinha cada uma das amigas?

3. Antônio estava contando seus pontos ao final de uma rodada de um jogo de cartas com seus amigos e resolveu lançar um desafio aos colegas. Ele propôs que se tivesse feito o triplo de seus pontos menos 37 pontos, teria o mesmo que o dobro de sua pontuação acrescido de 56 pontos. Quantos pontos marcou Antônio nessa rodada?

4. Uma balança de dois pratos está em equilíbrio. Em um prato há três pesos iguais de valor desconhecido (medido em gramas) e um quarto peso de $13 \mathrm{~g}$. No outro prato da balança há um outro exemplar igual aos anteriores de peso desconhecido e um peso de $45 \mathrm{~g}$. Qual é o valor do peso desconhecido?

\section{Quadro 1- Problemas apresentados na TRPEA Fonte: Sperafico (2013)}

Os problemas da TRPEA apresentam situações em que a tradução do problema verbal para uma equação algébrica é literal (problema 3), situações de tradução não literal (problema 1), situação com duas variáveis de valor equivalente (problema 2) e situações envolvendo a balança, que exigem maior reflexão para a compreensão das relações entre as grandezas. Entretanto, todos os problemas envolvem apenas operações no conjunto dos números inteiros.

Como recurso para quantificar os dados qualitativos obtidos pela tarefa de resolução de problemas (TRPEA) foram atribuídos valores às possibilidades de resultados da ação de resolução desempenhada pelo aluno, a saber: acerta (5 pontos); acerta após auxílio inicial (4 pontos); erra (3 pontos); erra após auxílio inicial ( 2 pontos) quando o aluno solicita auxílio para compreender o problema e não o soluciona corretamente; desiste (1 ponto) quando o aluno se julga incapaz de solucionar o problema.

\section{Resultados e discussão}


Os estudantes apresentaram no WASI uma média de 9,90 acertos ( $\mathrm{DP}=3,11)$ em um universo de 22 problemas. O desempenho entre os estudantes variou entre o mínimo de 5 (2DP) e máximo de 16 acertos (+2DP).

De acordo com o desempenho no WASI, os estudantes foram divididos em dois grupos: 1) grupo de alto nível de competência cognitiva (ACC) e 2) grupo de baixo nível de competência cognitiva (BCC). A designação dos estudantes para os grupos foi realizada com base na média geral do teste $(9,90$ acertos), sendo que 18 alunos com desempenho inferior à média compuseram o grupo $2(\mathrm{BCC})$ e 20 estudantes com desempenho superior à média compuseram o grupo 1 (ACC).

Investigaram-se também, por meio desse instrumento, as categorias de problemas do WASI em que os estudantes apresentaram melhor e pior desempenho. Os dois grupos demonstraram pior desempenho na categoria de Problemas de Resolução Matemática, já que aproximadamente $92 \%$ dos estudantes não apresentaram nenhum acerto nos problemas dessa categoria. No grupo 1, apenas três estudantes responderam corretamente um dos dois problemas de Resolução Matemática. Já no grupo 2, não houve acertos nos problemas dessa categoria. A categoria em que ambos os grupos apresentaram melhor desempenho foi a categoria de Relações de Frases Escritas, sendo que apenas dois estudantes (um de cada grupo) não responderam corretamente o problema que representava essa categoria.

Os problemas de resolução matemática, como propõe Mayer (1992), estão relacionados a conhecimentos linguísticos, factuais, de esquemas, de estratégias e algorítmicos. No caso dos estudantes do grupo 2, a baixa capacidade verbal (menos de 50\% de acertos, ver Gráfico 1) poderia ser uma das justificativas para o baixo desempenho nessa categoria. Entretanto, problemas em outros tipos de conhecimento envolvidos também podem ter acarretado dificuldades nos problemas matemáticos, como: conhecimentos factuais (compreender que um sexto é representado pela fração 1/6, como requeria um dos problemas da categoria); conhecimento do esquema (para identificar que o outro problema envolvia a relação da grandeza distância entre dois objetos distintos); conhecimento de estratégias para pensar em um plano de resolução (6:1/6, para descobrir quantos sextos há em 6) e conhecimento de algoritmo para executar o procedimento adotado (uma divisão de frações, no primeiro problema e uma regra de três no segundo).

No grupo 1, $80 \%$ dos estudantes também apresentaram desempenho insuficiente nos problemas matemáticos, apesar de, em sua maioria, terem demonstrado uma boa capacidade verbal. Isso pode sugerir que as dificuldades encontradas por esses estudantes possam ter 
origem em dois fatores ou em ambos: conceitos malformados em relação aos conhecimentos numéricos básicos necessários para a resolução de problemas matemáticos ou o conteúdo dos problemas (divisão de frações e regra de três) ainda não está dominado por esses alunos.

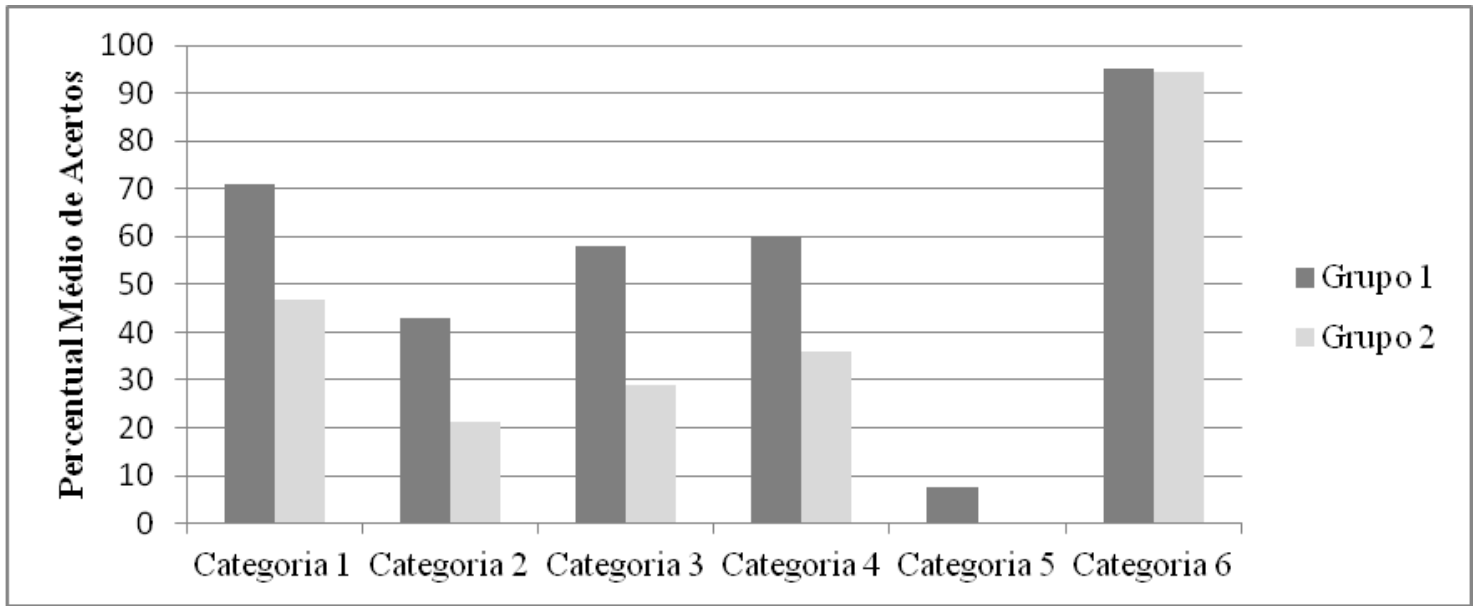

Gráfico 1- Desempenho geral dos grupos em cada categoria da Prova WASI Fonte: dados da pesquisa

Legenda: Categoria 1) Problemas de Raciocínio Verbal, Categoria 2) Problemas de Instruções Sequenciais, Categoria 3) Problemas de Formação de Analogias, Categoria 4) Problemas de Análise de Tendências e Padrões, Categoria 5) Problemas de Resolução Matemática e Categoria 6) Problemas de Relação de Frases Escritas.

Como se pode constatar no Gráfico 1, o grupo 1 demonstrou um desempenho mais homogêneo em relação às categorias (exceto a categoria de Problemas Matemáticos, já comentada), não havendo, na maior parte dos casos, predomínio absoluto de uma categoria em detrimento de outras. Já o desempenho dos estudantes do grupo 2 foi mais heterogêneo, pois grande parte dos alunos apresentou um desempenho melhor na categoria Relações de Frases Escritas, mas baixo desempenho nas demais. Entretanto, cabe destacar que muitos estudantes desse grupo apresentaram baixo desempenho em todos os tipos de problema.

Em relação à Tarefa de Resolução de Problemas com Equações algébricas do $1^{\circ}$ grau (TRPEA), os estudantes apresentaram uma pontuação média de 15,71 pontos ( $\mathrm{DP}=2,55)$, com máxima de 20 pontos (+2DP) e mínima de 10 pontos (-2DP), demonstrando, em primeira análise, um bom desempenho na resolução dos problemas. O desempenho médio em cada questão, por grupos, pode ser verificado na Tabela 1, em que se observa um melhor desempenho dos estudantes do grupo 1 (ACC) na resolução dos problemas da TRPEA (lembramos que a pontuação máxima é de 5 pontos em cada problema).

Tabela 1- Desempenho médio dos estudantes de cada grupo nos problemas matemáticos

\begin{tabular}{llcccc}
\hline & & Problema 1 & Problema 2 & Problema 3 & Problema 4 \\
\hline \multirow{2}{*}{ Grupo 1 } & Média & 3,83 & 4,56 & 4,72 & 4,22 \\
& DP & 0,79 & 0,51 & 0,57 & 0,55
\end{tabular}




\begin{tabular}{lllccc} 
& Média & 2,44 & 3,67 & 4 & 3,67 \\
Grupo 2 & DP & 1,15 & 0,91 & 0,84 & 0,84 \\
\hline \multicolumn{5}{c}{ Fonte: dados da pesquisa }
\end{tabular}

Os resultados do WASI apresentaram correlação estatisticamente significativa $(0,638$; p<0,001) com a TRPEA (apresentação da distribuição dos dados no Gráfico 2), mostrando que há relação entre a competência cognitiva mais geral para a resolução de problemas e o desempenho na resolução de problemas com equações algébricas do $1^{\circ}$ grau.

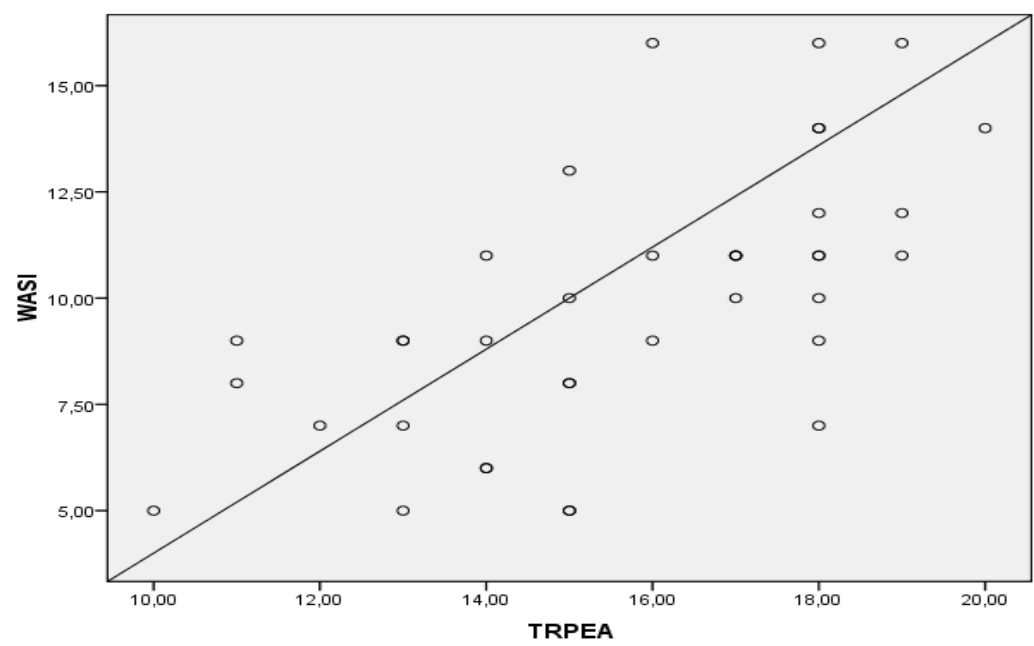

Gráfico 2- Correlação entre a Prova WASI e a TRPEA

Fonte: dados da pesquisa

A aplicação do teste t-Student também evidenciou diferença significativa entre os grupos em relação à TRPEA $(5,687 ; \mathrm{p}<0,001 ; \mathrm{M}=3,46 ; \mathrm{DP}=0,61)$.

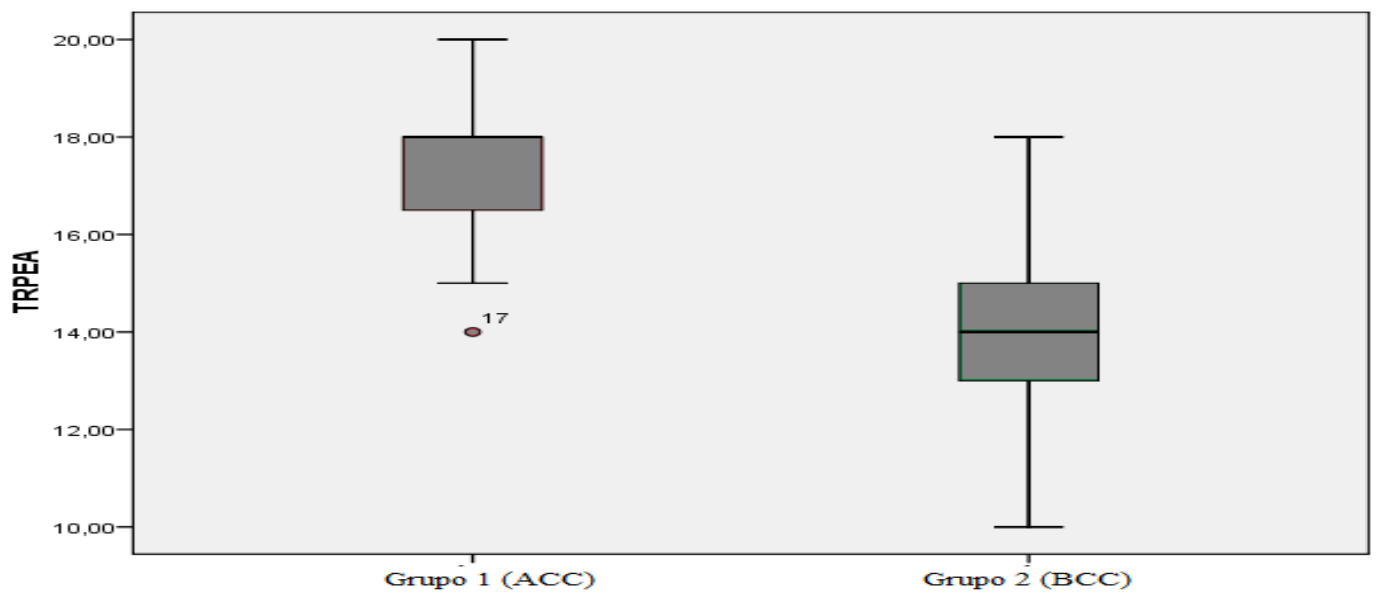

Gráfico 3- Diferença entre os grupos para a TRPEA Fonte: dados da pesquisa

Pode-se observar, no Gráfico 3, que no grupo 1 há um ponto que representa um dado do grupo que está abaixo do limite inferior da amostra, ou seja, um sujeito do grupo 1 que 
teve um desempenho bastante inferior à média do grupo. No grupo 2 há um desempenho bastante heterogêneo, como pode ser verificado pelo comportamento do intervalo apresentado no gráfico, sendo que alguns estudantes apresentaram desempenho semelhante aos estudantes do grupo 1 .

Essa diferença estatisticamente significativa entre os grupos 1 (ACC) e 2 (BCC) sugere que alunos com maiores níveis de competência cognitiva apresentam melhor desempenho na resolução de problemas com equações algébricas do $1^{a}$ grau. Esse achado corrobora os estudos de Chi e Glaser (1992), Nokes, Schunn e Chi (2010) e Chi (2011) que sugerem que diferenças no desempenho estão baseadas em processos cognitivos e organizações mentais, sendo que alunos mais competentes possuem maior capacidade de organização do conhecimento na memória de longo prazo, podendo acessá-lo durante a resolução do problema; processam melhor as informações envolvidas na resolução; possuem esquemas ricos e organizados; e utilizam processos de automatização de etapas da resolução do problema que possibilitam pouco controle consciente, prevenindo a sobrecarga da memória de trabalho, o que resulta em melhor desempenho.

\section{Considerações Finais}

O presente estudo destacou a existência de correlação entre a competência cognitiva geral para a resolução de problemas e o desempenho na resolução de problemas com equações algébricas do $1^{\text {o }}$ grau, evidenciando um melhor desempenho em estudantes mais competentes. Tal diferença no desempenho resulta de conhecimento conceitual e processual bem organizado e processos cognitivos estruturados, que permitem um melhor monitoramento da resolução. Desse modo, na área de resolução de problemas, parece haver possibilidade de generalização de uma capacidade mais ampla para situações mais específicas, como é o caso dos problemas matemáticos com equações algébricas do $1^{\circ}$ grau.

Cabe, ainda, destacar que há limitações que impossibilitam a generalização desse resultado, como a amostra reduzida, a abordagem de somente duas variáveis e a TRPEA que possui número reduzido de problemas para avaliar o desempenho. Contudo, este estudo tem implicações para o ensino, pois, ao apontar a relação direta entre competência cognitiva e desempenho na resolução de problemas envolvendo equações algébricas do $1^{\circ}$ grau, evidencia a importância dos processos cognitivos que estão subjacentes à resolução de problemas e que podem ser potencializados pelos estudantes, por meio da prática de estratégias que podem ser 
trabalhadas em sala de aula. Um ensino voltado também para o desenvolvimento de capacidades cognitivas, e não apenas para o exercício do conteúdo matemático, é provável de ser eficaz no que se refere à resolução de problemas com equações algébricas do $1^{\circ}$ grau. Entretanto, há necessidade de mais estudos, incluindo estudos interventivos, a fim de verificar a possibilidade do ensino de estratégias cognitivas que visem o aprimoramento da competência cognitiva e o seu efeito na aprendizagem desse e outros conteúdos matemáticos.

\section{Referências}

BERNARD, J.; COHEN, M. Uma integração dos métodos de resolução de equações numa sequência evolutiva de aprendizado. In: COXFORD, A.; SHULTE, A. (Org.). As ideias da álgebra. Tradução: Hygino Domingues. São Paulo: Atual, 1995. p. 111-126.

BOOTH, L. Dificuldades das crianças que se iniciam em álgebra. In: COXFORD, A.; SHULTE, A. (Org.). As ideias da álgebra. Tradução: Hygino Domingues. São Paulo: Atual, 1995. p. 23-36.

BOOTH, J.; KOEDINGER, K. Key misconceptions in algebraic problem solving. In: LOVE, B.; MCRAE, K.; SLOUTSKY, V. (Ed.). Proceedings of the 30th Annual Cognitive Science Society. Austin: Cognitive Science Society, 2008. p. 571-576.

CAMPOS, R. Estudo comparativo das habilidades motoras e cognitivas em praticantes de futebol de diferentes locais de prática. 2004. 121 f. Dissertação (Mestrado em Educação Física) Programa de Mestrado do Departamento de Educação Física do Setor de Ciências Biológicas, Universidade Federal do Paraná, Curitiba, 2004.

CHASE, W.; SIMON, H. Perception in chess. Cognitive Psychology, v. 4, n. 1, p. 55-81, jan. 1973.

CHI, M. Theoretical Perspectives, Methodological Approaches, and Trends in the Study of Expertise. In: LI, Y. (Ed.). Expertise in Mathematics Instruction: an international perspective. New York: Springer, 2011. p.17-39.

CHI, M.; GLASER, R. A capacidade para a solução de problemas. In: STERNBERG, R. As capacidades intelectuais humanas. Porto Alegre: Artmed, 1992. p. 250-275.

CHI, M.; FELTOVICH, P.; GLASER, R. Categorization and representation of physics problems by experts and novices. Cognitive Science, v. 5, n. 2, p. 121-152, apr. 1981.

CHI, M.; ROY,M.; HAUSMANN, R. Observing tutoring collaboratively: Insights

about tutoring effectiveness from vicarious learning. Cognitive Science, v. 32, n. 2, p. 301-341, mar. 2008.

DA PONTE, J. P.; BRANCO, N.; MATOS, A. Álgebra no Ensino Básico. Portugal: Ministério da Educação-BGIdc, 2009. p. 92-115.

DANTE, L. R. Equações do $1^{\circ}$ grau com uma incógnita. In: DANTE, L. R. Tudo é matemática $6^{\mathbf{a}}$ série. 2ed. São Paulo: Ática, 2007. p. 99-124.

DAVIS, C.; NUNES, M. M. R.; NUNES, C. A. A. Metacognição e sucesso escolar: articulando teoria e prática. Cadernos de Pesquisa. São Paulo, v. 35, n. 125, p.205-230, mai./ago. 2005. 
DE GROOT, A. Perception and memory versus thought: Some old ideas and recent findings. In: KLEINMUNTZ, B. (Org.). Problem solving: Research method and theory. New York: Wiley, 1966. p. 19-50.

DUFRESNE, R.; GERACE, W.; HARDIMAN, P. T.; MESTRE, J. P. Constraining novices to perform expert like problem analyses: Effects on schema acquisition. Journal of the Learning Science, Mahwah, v. 2, n. 3, p. 307-331, jul. 1992.

GINSBURG, H. The challenge of formative assessment in mathematics education: children's minds, teacher's minds. Human Development, Basel, v. 52, n. 2, p. 109-128, apr. 2009.

GULGOZ, S.; KAGITCIBASI, C. Intelligence and intelligence testing in Turkey. In: STERNBERG, R. (Ed.) International Handbook of Intelligence. Nova York: Cambridge University Press, 2004. p. 248-269.

MATLIN, M. Resolução de problemas e criatividade. In: MATLIN, M. (Ed.). Psicologia Cognitiva. Rio de Janeiro: LTC, 2004. p. 233-259.

MAYER, R. A capacidade para a matemática. In: STERNBERG, R. (Org.). As capacidades intelectuais humanas. Porto Alegre: Artmed, 1992. p.114-168.

NOKES, T.; SCHUNN, C.; CHI, M. Problem solving and human expertise. In: BAKER, P.; MCGAW, B. (Ed.). International Encyclopedia of Education. 3ed. Oxford: Elsevier, 2010. p. 265272.

OECD. PISA 2012 field trial problem solving framework: Draft subject to possible revision after the field trial. Paris: OECD, 2010. Disponível em: $<\mathrm{http} / / / w w w . p i s a$. oecd.org/dataoecd/8/42/46962005.pdf>. Acesso em: 10 out. 2012.

PRIMI, R.; SANTOS, A. A. A.; VENDRAMINI, C. M.; TAXA, F.; MULLER, F. A.; LUKJANENKO, M. F.; SAMPAIO, I. S. Competências e habilidade cognitivas: diferentes definições dos mesmos construtos. Psicologia: Teoria e Pesquisa, Brasília, v. 17, n. 2, p. 151-159, mai./ago. 2001.

PRIMI, R. Inteligência fluída: definição fatorial, cognitiva e neuropsicológica. Paidéia, Ribeirão Preto, v. 12, n. 23, p. 57-75, jan. 2002.

REINERT, C. A preliminary comparison between two methods of intellectual skill development. In: CONFERENCE OF THE SOCIETY FOR ACCELERATIVE LEARNING AND TEACHING, 1989, San Diego. Proceedings... San Diego, apr. 1989.

SANLEY, J. D. Na examination of student learning styles and learning modalities on problemsolving success. Lincoln: ETD collection for University of Nebraska, 1987.

SANTOS, M. A.; PRIMI, R. Desenvolvimento de um teste informatizado para a avaliação do raciocínio, da memória e da velocidade do processamento. Estudos da Psicologia, Campinas, v. 3, n. 22, p. 241-254. jul./set. 2005.

SPERAFICO, Y. L. S. Competências cognitivas e metacognitivas na resolução de problemas e na compreensão do erro: um estudo envolvendo equações algébricas do $1^{\circ}$ grau com alunos do $8^{\circ}$ ano. 2013. 153 f. Dissertação (Mestrado em Educação) - Programa de Pós Graduação em Educação, Faculdade de Educação,Universidade Federal do Rio Grande do Sul, Porto Alegre, 2013.

TARDIF, J. Développer un programme par compétences: de l'intention à la mise en oeuvre. Pédagogie collégiale, Québec, v.16, n. 3, p. 36-44, mar. 2003. 
VERGNAUD, G. The theory of conceptual fields. In: STEFFE, L.; NESHER, P.; COBB, P.; GOLDIN, G.A.; GREER B. (Eds.). Theories of mathematical learning. Mahwah: Lawrence Erlbaum Associates, 1996. p. 219-239.

VIEIRA, E. Intervenção psicopedagógica na fase de representação mental em resolução de problemas matemáticos. 1999. 206 f. Tese (Doutorado em Psicologia) - Programa de Pós-Graduação em Psicologia, Pontifícia Universidade Católica do Rio Grande do Sul, Porto Alegre, 1999.

WHIMBEY, A.; LOCHHEAD, J. Problem solving and comprehension. Mahwah: Lawrence Erlbaum Associates, 1999.

Submetido em Abril de 2014. Aprovado em Julho de 2014. 